\title{
EAl Endorsed Transactions

\section{A review of youth mental health promotion apps towards their fit with youth media preferences}

\author{
T. Michel ${ }^{1, *}$, F. Tachtler ${ }^{1}$, P. Slovak ${ }^{2}$, and G. Fitzpatrick ${ }^{1}$ \\ ${ }^{1}$ Department of Informatics, TU Wien, Vienna, Austria \\ ${ }^{2}$ Department of Informatics, King's College, London, UK
}

\section{Abstract}

INTRODUCTION: Mental health promotion apps can promote youth mental health but fail to engage young people. Fit to young people's media preferences is known to mediate engagement.

OBJECTIVES: To explore the fit of existing youth mental health apps with young people's media preferences.

METHODS: A workshop with 60 youth psychologists elicits designs of digital mental health interventions. A review of 29 youth mental health apps unpacks their modality strategies. We then compare modality strategies from literature on youth mental health, media preferences and engagement, and from the experts, with strategies in current mental health apps to identify potential fit problems.

RESULTS: There is a mismatch between young people's modality preferences and how youth mental health apps deliver their content.

CONCLUSION: There is a need to make youth mental health promotion apps more interactive and tailorable, featuring dynamic visuals and social connectivity, to better engage youth.

Keywords: Healthcare technology, Patients' needs, Applications

Received on 23 November 2018, accepted on 29 January 2019, published on 12 February 2019

Copyright (C 2019 T. Michel et al., licensed to EAI. This is an open access article distributed under the terms of the Creative Commons Attribution licence (http://creativecommons.org/licenses/by/3.0/), which permits unlimited use, distribution and reproduction in any medium so long as the original work is properly cited.

doi: 10.4108/eai.13-7-2018.161419

"Corresponding author. Email: toni.michel@tuwien.ac.at

\section{Introduction}

Up to $70 \%$ of children and adolescents with mental health problems do not receive appropriate care at a sufficiently early age [1]. Mental health problems during youth (10 to 24 years of age [2]) often persist into adulthood [3]. This is not only economically disastrous-e.g. the UK economy loses an estimated $£ 105$ billion a year due to mental health problems [4] - but also takes a human toll. Suicide has become the most common cause of death for boys between 15 and 19 years of age; for girls it is the second most common cause of death [5]. These trends need to be countered by facilitating early access to mental health promotion and prevention measures [6].

Recent evidence suggests that prevention and promotion interventions are the most efficient ways to improve population-level mental health [7]. However, even though young people represent the age-group most likely to develop mental health problems, they are also least likely to actually receive help [7].

This gap-between needing and receiving support - is mainly a consequence of two issues: society stigmatizing mental health problems [7] and overstretched mental health services which mean young people are on waiting lists for weeks and months [8]. Stigma stifles help-seeking behavior due to fear of social shaming [7], and when young people do reach out for help, often they still do not receive it in time [8]. 
Mental health experts advocate that technology may help alleviate pressure on services and offer a stigma-free avenue for support [9]. Howeverdespite evidence for the effectiveness of various mental health technologies [10], [11]—recent research has revealed that there is low uptake, adherence and engagement of young people with mental health technology [13], [8].

Promotion and prevention apps - the area addressed by this paper-aim to provide young people with activities, peer-support and information [14], to improve their wellbeing and resilience, leading to a better quality of life and a decreased risk of pathologies manifesting respectively [15]. It follows that use of these apps could also potentially reduce the current strain on mental health services by freeing up services to focus on providing psychologist or therapist support for youth with more pronounced needs [9].

Whether or not young people pick up a mental health activity - and whether or not they stick with it - is partially predicated on how well the activity fits them [16]. One prominent model for conceptualizing this fit is the Person-Activity Fit (PAF) [17] where fit is an emergent property between the features of an activity, such as variety and dosage, and individual differences, such as motivation, personality, and demographics [17], [18]. The better these align, the better the PAF, and the higher the likelihood of initial and prolonged engagement with an activity [17].

The issue of engagement with digital youth mental health interventions - and how to design to promote engagement-has been identified as needing much more research [19]. Further, the construct of "engagement" has no generally agreed upon definition. In research, terms and phrases to describe aspects of engagement include: attraction, playfulness, sensory integration, usability, enjoyment, as well as "media presentation, perceived user control, choice, challenge, feedback, and variety" [20]. In the early 1990s, engagement had already been identified as "a desirable - even essential-human response to computer-mediated activities" [21], and it may have become even more relevant since then [20]. O'Brien and Toms define engagement as: "a quality of user experience with technology that is characterized by challenge, aesthetic and sensory appeal, feedback, novelty, interactivity, perceived control and time, awareness, motivation, interest, and affect" [20]. We concur with O'Brien and Tom and see engagement as rooted in the characteristics they stated, which do align with our findings presented in this paper.

However, to date there has been no study into how these qualities are interpreted in youth mental health promotion apps to ensure a fit with young people. Given that fit mediates engagement, and that overall engagement with youth mental help apps is low, we pose the following question: Could low engagement with youth mental health promotion apps be explained by a lack of fit between youth media preferences and established digital intervention delivery methods?

We explored this question through the following steps:

(i) An explorative workshop with 60 mental health experts-i.e., active members of the British Psychological Society (BPS) - to establish strategies to achieve fit (see Study 1: Expert perspective)

(ii) A review of 29 existing youth mental health prevention and promotion apps to understand how they deliver content (see Study 2: App review)

(iii) A comparison of (1) and (2) with elements which the research suggests young people prefer in apps, to establish the degree to which they converge or diverge (see Discussion and limitations)

What we found suggests a misalignment between how youth mental health promotion apps deliver their content and the strategies of fit we established in Study 1 in conjunction with our initial literature review (see Related work).

In the following section, we will connect our review with a broader research context, then explain our qualitative, constructivist methodology. We will then explicate in detail how we applied a Thematic analysis (Study 1) and General inductive approach (Study 2), which led us to discover a gap between how youth mental health promotion apps present their content, and how young people are likely to prefer to receive it. We then discuss how this impacts a broader research context by arguing for the additional benefits of designing in line with young people's preferences beyond potentially increasing engagement. Finally, we offer some implications from our findings, most notably that it may be necessary to change how we design youth mental health promotion apps, and potentially, by extension, youth mental health technology in general, in order to make them more interesting, more engaging, and more intrinsically rewarding for young people to use.

\section{Related work}

In this paper, we address a multidisciplinary research question and, in order to do so, we draw on research into youth mental health, as well as human-computer interaction (HCI). We start by summarizing the current mental health technology research, and then focus on research into mental health apps specifically. Following this, we expand on the concept of fit and present a specific model through which fit may be conceptualized. We then 
ssintroduce the current literature on youth media preferences, both within and beyond a mental health context. Finally, we introduce the concept of technological multimodality (MM) and identify why $\mathrm{MM}$ is a suitable lens through which to conduct this research.

Our use of the terms "prevention" and "promotion" in this paper is applied from a Positive Psychology (PP) perspective [22], [23], which considers prevention as a benefit that is achieved through promotion, while also recognizing that prevention and promotion are interconnected and mediate each other [22]. The term "prevention" is also used in a clinical context, in which it is "characterized by a sense of obligation and vigilance against potential loss" [24]. This differs from our use of the term through its focus on avoiding pathology. In contrast, we view prevention as a benefit derived from building strengths [22].

\subsection{Technology for mental health}

\section{General technology for mental health}

Young people are open to using mental health technologies, and there is data to suggest that some mental health technologies produce positive outcomes. For example, a survey of 922 university students aged between 18 and 24 showed an overwhelming willingness to use internet-based mental health services, and also demonstrated that about one in three students had already made use of such services in the past [25]. There have also been a number of studies on the effectiveness of some digital mental health services. For example, a systematic review of 52 randomized-controlled trials (RCT) showed strong evidence for the effectiveness of internet-based Cognitive Behavioral Therapy (CBT) [26]. This held true for a meta-analysis of 13 RCTs, which, overall, included 796 children and adolescents, exploring the effectiveness of internet-based CBT specifically for young people [27]. Additionally, the Mental Health Commission of Canada conducted a literature review spanning 261 studies on different types of mental health interventions and technologies from the UK, Europe, Australia/New Zealand, Scandinavia, the USA, Asia/Middle East and Canada and found that 215 out of 261 studies showed "statistically significant positive change on primary outcomes" [28], reflecting that there are working technology-enabled mental health interventions.

\section{Mental health apps}

While the above-mentioned studies focus on internet-delivered interventions, reliable studies into the efficacy and effectiveness of, specifically, mental health apps are rare, and the results are contradictory. A 2013 systematic review of mental health apps was only able to identify five apps related to studies which either had pre- to post-test designs, or used a control group [29]. For those five apps, effectiveness was confirmed. Only two of them were publicly available for download, and only one of them offers an English version: $D B T$ Coach; it aims to offer Dialectical Behaviour Therapy (DBT) to adults suffering from borderline personality disorder. Meanwhile, a 2017 systematic review on the efficacy and acceptability of mental health apps for children and adolescents could not arrive at any positive findings on effectiveness [30]. Gist et al. reviewed 24 publications. The 15 apps studied in these 24 publications ranged from prevention and early intervention, to assessment and screening, to adjuncts in face-to-face mental health care, as well as standalone self-help interventions. While they could confirm acceptability, Gist et al. conclude that there is insufficient evidence to support effectiveness [30].

Following an alternative line of inquiry, a 2016 review of mental health apps proposed a list of 16 recommendations to aid developers by summarizing research on specific components of mental health apps, e.g. the ability to track emotions [31]. While looking at components would arguably provide a more useful level of granularity, they too, like both previously mentioned reviews, stressed that available data is very limited, and that generalizable statements on effectiveness and efficacy cannot yet be made [31].

Still, various large mental health organizations, e.g. ReachOut Australia and the Anxiety and Depression Association of America [32], [33], have started recommending mental health apps, despite a lack of definite evidence on their overall effectiveness. Both offer curated lists which report, among other things, the evidence-base for individual apps, by rating them between 1 and 5 . While this rating allows a quick overview, it is unclear what criteria have been used for it. It is also not possible to search for apps based on personal preferences, for example, if trying to find a gamified approach to Gratitude exercises.

Overall, there is a range of available digital mental health services, including apps, used across the world, with a growing (but still limited) evidence-base to support their effectiveness, and major non-governmental organizations in the mental health sector signposting them to potential users, including young people. There is also a persistent lack of rigorous research on all aspects of these technologies.

\subsection{The role of fit}

One of these aspects is around fit, as mental health research increasingly moves from asking "What works?" to "What works for whom?" [17]. The better an activity aligns itself with a person's 
individual preferences and interests, the more intrinsically motivated that person is to engage with the activity, and the more intrinsically motivated, the more time and effort are invested, leading to better outcomes [11], [20]. It is for this reason that fit has been recognized as "critical to the efficacy" of mental health interventions [18].

One widely-used model to describe this type of fit specifically in the context of PP is PersonActivity Fit (PAF) by Layous and Lyubomirsky [35]. This model describes the interplay between the features of an activity and the features of a person, and how this interplay translates into wellbeing gains.

While fit impacts all types of mental health interventions, it is currently given the most consideration within PP [17]. Schueller suspects that the reason for this lies in the relatively low complexity and brief nature of PP activities, which can, predominantly, be completed in under four weeks. This allows them to be used as interchangeable kernels instead of complex, overarching approaches, like psychotherapy, where it would be more difficult to individualize the approach in a systematic, evidence-based way [17].

\subsection{Youth preferences}

As our goal was to review youth mental health prevention and promotions apps from the perspective of fit, we also explored the literature on youth preferences, both inside and outside the mental health context. The general media preferences of young people are continuously studied by market research, and this means that large-scale studies on the issue are available. Studies on youth preferences with regard to mental health technology, however, are comparatively rare. Still, a review of what this work suggests regarding youth preferences provided us with a useful basis from which to position the findings from our own research.

\section{General preferences}

One clear finding is the prevalence of mobile devices among young people. The Ofcom 2017 Report on Children and Parents: Media use and Attitudes [36] reports that $93 \%$ of children aged 1215 own a smartphone. More than half of children in the same age group already own a tablet. These devices are used for surfing the internet, watching $\mathrm{TV}$, interacting socially and playing games-a slight gender difference showing that girls favor use for social interaction and boys for playing games. Smartphone ownership increases rapidly from $76 \%$ at 13 to $92 \%$ at 14 , and then seems to level off at age 15 at $93 \%$. This shows a convergence towards almost every young person owning a smartphone [36].
There are also indicators of increasing preference for more social and interactive genres of use. For example, the Ofcom report shows a rapid shift in the social media landscape; in 2013, $87 \%$ of children aged 12-15 used Facebook as their main social messaging service, and only $40 \%$ of children in 2017. At the same time, Snapchat, which did not exist in 2013, served as the main messaging service for $16 \%$ of children in 2016 and 32\% in 2017 [36]. It is worth noting that Snapchat is a much more visually driven platform compared to Facebook. The report also points out that young people increasingly gravitate away from passive media like TV and towards interactive types of media, like social media and games [36]. Related to this, a 2015 US survey of 3,279 children and young people aged 2-17 shows that $92 \%$ of them play video games on a regular basis [37]. A connected 2018 genre break-down of game sales for the US shows that action games and shooter games account for $47.8 \%$ of games sold, making these inherently fast-paced, highly interactive games the most favored genres [38].

\section{Mental health preferences}

We can compare these findings to media preferences documented in a mental health context. While the data here is not of the same size and strength as provided in a general context, they largely affirm the aforementioned trends. A 2014 scoping review by Boydell et al. collected and reviewed 126 original studies on the use of technology to deliver mental health services to young people [39]. They found that young people consistently prefer highly interactive and customizable content, and strongly dislike static content, i.e., static text and images, in a mental health context.

Young people especially want the option of social interaction, i.e., social media mechanics, allowing them to interact with both experts and peers. When given the choice about how to interact with a mental health expert, young people favor video conferencing over in-person meetings [39]. A 2016 study by Fleming et al. on adolescents' attitude towards computerized therapy indicates that young people especially prefer to receive interventions through games, as long as these games are of sufficiently high quality [40].

In summary, existing research indicates that general youth media preference trends apply in a mental health context - that young people prefer content to be delivered through mobile devices, that is highly interactive and driven by dynamic visual media, e.g., games, and that is flexible so that it can be adjusted to their individual needs.

\subsection{Technological multimodality}


Much of this interactivity can be interpreted through the lens of multimodality (MM). MM is the practice of offering multimodal output and/or multimodal input. A modality describes an elemental type of input or output which a person gives or receives from a system [41]. Multimodality refers to the interplay between, as well as emergent qualities of, using more than one modality for input and output at a time [41]. It may describe having a choice of modality for the person interacting with a system, or the process of synthesizing multiple modalities in order that they work better together. MM has been established in HCI research as a way to make systems more tailorable, more efficient, more usable, more accessible, with reduced errorproneness, and with the opportunity for increased immersion and hedonic qualities, for example through allowing deeper involvement of the body [41]-[44]. With its impact on user-experience, novelty, interactivity and control, MM is closely connected with the notion of engagement as used in this paper.

Despite its proven relevance to HCI and utility as an exploratory lens through which to study digital interactive systems, MM has not yet been used as a primary perspective from which to study mental health technology with a view to engagement and fit. Most studies describing mental health technologies either omit the modalities they employ, or they mention them inconsistently and with little specificity. The most attention given to modalities in mental health apps, among all the papers we were able to find, was a 2015 review of depression apps by Shen et al. [45]. Shen et al. linked common modalities with treatment approaches, for example the prevalence of audio in therapeutic treatment apps. They also reported that the majority of depression apps exclusively relied on text to deliver their content - a potential issue that foreshadowed much of what we ended up identifying in our app review.

\subsection{Summary}

We draw from youth mental health and HCI research in order to explore our research question: Could a lack of fit between youth media preferences and digital intervention delivery practices explain the observably low rates of uptake and engagement of youth mental health apps? Our literature review, outlined in this section, shows the abundant potential of technology to promote mental health in young people. A growing body of research documents the effectiveness of mental health technology in general, and especially of apps for mental health promotion, while also observing low rates of uptake and engagement with these technologies. Across disciplines, research increasingly documents factors relevant to engagement. In psychology, the recent notion of person-activity fit suggests that fitting interventions to individual preferences impacts people's willingness to engage with these activities. In HCI, the notion of technological multimodality is increasingly used to explain people's willingness to engage with technology, as well as the experiences technologies facilitate. If considered side by side, these strands of research offer a pathway to explain and remedy the issue of low engagement with youth mental health technologies. Yet, up until now, they have not been connected. In doing so, we discovered both a potential reason for low engagement, as well as a way forward, rooted in improving fit and multimodality as pivotal aspects of youth mental health technology.

\section{Methodology}

To explore these issues, we follow a qualitative, constructivist approach [46] in our research, with an ultimate view to understanding how we might design mental health apps that better fit with young people. Thus, the data we collect, and the constructs we build from it, are "socially and experientially based, local and specific in nature" [47]. We assume that there is a link between the object of our research and the researcher, and that, therefore, our findings are "literally created" [47] during this process. Our goal is to provide a construct that is more informed and sophisticated than existing constructions [47]-i.e., to provide the strongest construct describing the current degree and shortcomings of fit between existing youth mental health apps and young people, to better design fitting mental health apps in the future. The construct we provide through this research - an exploration of the relation between youth media preferences and technological intervention delivery practices-is inherently transitional and will be replaced in time by a more sophisticated and/or more current one [47]; until then, it will provide utility for the design of youth mental health promotion apps and closely related technologies.

We performed two studies using the aforementioned approach to explore our question of whether the way youth mental health promotion apps deliver their content may contribute to the low engagement of young people with these apps.

Study 1 asks 60 youth mental health experts how they would design engaging mental health technologies, drawing on their experience of working with diverse young people across the UK; this study is conducted in a workshop setting. A thematic analysis [48] of their concepts and discussions during the workshop pointed to shared strategies to make these technologies engaging for young people, e.g., highly interactive content. 
Study 2 builds on this and reviews youth technology preferences by considering the strategies used in 29 publicly available youth mental health prevention and promotion apps to deliver their interventions, pointing to an underuse of diverse modalities, e.g., a heavy reliance on text and static imagery. We arrived at these strategies by creating rich descriptions of the apps and subsequently analyzing these descriptions using a general inductive approach [49].

We now walk through each study and its findings in turn.

\section{Study 1: Expert perspective}

In Study 1, we explored the perspective of youth mental health experts in creating engaging mental health technologies.

Even though our exploration of relevant literature provided us with some candidate strategies for fit with youth preferences, it was not obvious that general media preferences would automatically translate into mental health technology needs. Additionally, most of the data on youth preferences for mental health technology which we were able to find (e.g., [50], [51]) looked specifically at the treatment-space, which may also come with its own set of strategies to achieve fit, and in turn, may not translate into effective mental health promotion. The strong overlap between both perspectives-general media preferences and mental health technology needs - was a promising start, but an additional exploratory step was necessary to define strategies for fit of which we were reasonably confident.

We thus conducted a one-hour long exploratory workshop with 60 youth mental health experts from the UK during a meeting of the BPS in London. The workshop was part of a regular meeting of the BPS. Attendees were informed in advance that the workshop would be taking place through the respective meeting proceedings. All attendees agreed to take part in the workshop. Prior to conducting this study, we also received ethical clearance from the University College London Research Ethics Committee (project 6087/011).

This group of youth mental health experts was able to draw from experiences working with large numbers of diverse young people across the UK, and in turn, arguably contribute a more general perspective than a single workshop with a few young people might have offered.

\subsection{Methods}

The workshop thus served as a scoping activity to gather professional opinions on promising strategies to create engaging youth mental health technologies, against the backdrop of our initial literature review. Our 60 participants were randomly assigned to nine different groups and then asked to develop ideas for youth mental health technologies. We did not specify an area to focus on, except that the idea should be aimed at mental health promotion, and that it should be engaging for young people, based on their professional opinions and experiences of working with young people. We did not prime them with any specific technologies or approaches we would consider suitable for this space; they were asked to work purely from their own experience. Two researchers facilitated the workshop: one clinical psychologist specialized in youth mental health, and one technology expert (the first author, taking notes and answering questions focused on the feasibility of ideas).

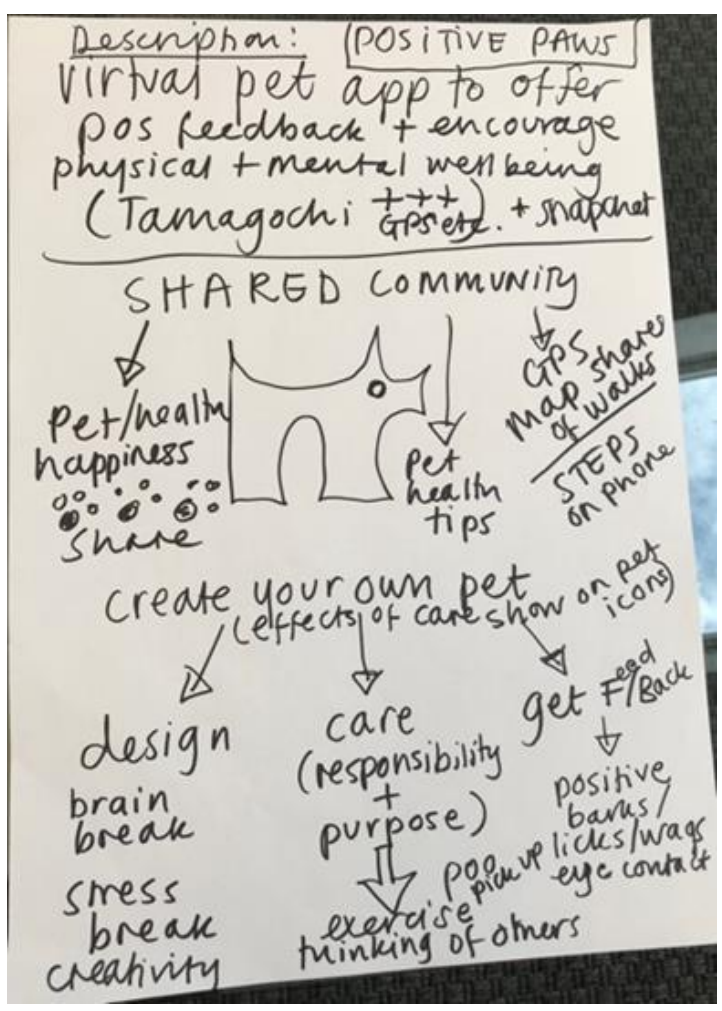

Figure 1. Group idea during expert workshop

After an initial opportunity for individual reflection, groups discussed potential directions and decided on one idea per group which they then developed further. The choice of idea was left to them. Towards the end of the workshop, each group presented their ideas to each other in the form of posters (see for example Fig 1; see Table 1 for a full list of the poster ideas by group, including the solution name and short description). Subsequently, there was a short discussion about each idea. We took notes during the group work, as 
well as the presentations and discussion, and collected the written summaries of ideas generated by each group. We then analyzed their strategies.

\subsection{Data analysis}

We were primarily interested in the technological strategies to deliver content. Using a thematic analysis approach [48], we first developed and applied a number of codes describing different types of strategies proposed in their ideas, for example tracking or avatar. We then analyzed the data iteratively to first find a coding scheme that captured the data set (see for example Fig 2) and then a set of themes to represent those codes.

Overall four researchers were part of the data analysis process to varying degrees (see Table 1). Codes and themes were developed primarily by the first author (R1). Results, coding and themes were discussed iteratively in collaborative review sessions with the other three researchers, to identify and address ambiguities, possible contradictions, and aspects of the data which seemed especially relevant.

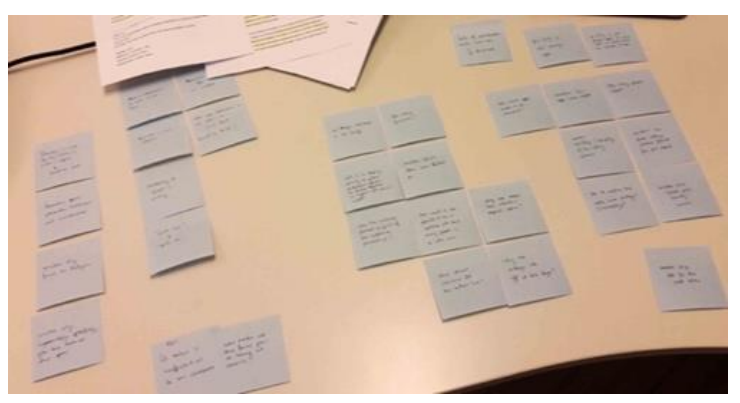

Figure 2. Intermediate step of thematic analysis

\subsection{Results}

As Table 1 shows, the developed concepts spanned a wide range of domains, from specific vulnerable groups, like diabetic children (Concept 2 ), to specific issues, like sleeping problems (Concept 5) or bullying (Concept 6), to specific strategies, like self-reflection (Concept 3). Even with this diversity, we were still able to identify repeated calls for certain types of functionality and technology attributes. They are as follow.

Theme 1: Facilitating social connectivity Concepts 1, 2, 5-8, and 9 emphasized social connectivity, especially in the form of peer networks. Our participants saw, in peers, both a source of information and a potential support structure. Other young people with lived experience could provide advice which had more authenticity than may be the case for a seemingly further-removed expert. Being able to provide advice to other struggling young people was also seen as a possible mechanism of empowerment, by moving the young person from a perceived 'victim' role to a supportive one.

They also stressed the importance of creating access to experts as a source of reliable information.

\section{Theme 2: Inclusion of video}

Concepts 1, 6 and 7 proposed the incorporation of videos as a way of providing information to young people. Our participants saw, in some cases, the potential of video as an additional way to make information accessible, alongside other modalities. They argued that some young people would be more willing to watch a short video than read the same information as static text. Also, if they are "on the go", then they could just listen to the audio, and would still be able to receive important pieces of information.

One concept also directly proposed creating a YouTube channel (see Concept 7). The aim of the channel would be to spread information, but also to allow prominent people to speak about their lived experiences with mental health issues. If prominent people were willing to share their lived experiences of mental health issues on a platform accessible and used by young people, then, it was felt, this would be helpful in reducing the prevailing stigma. It was also noted that making use of already existing technologies and infrastructures like YouTube would both allow services to be offered on a more realistic budget, and meet young people in a forum they already use.

Table 1. Researchers involved in data analysis

\begin{tabular}{|c|c|c|}
\hline \# & Qualification & Role \\
\hline $\mathrm{R} 1$ & $\begin{array}{l}\text { Early stage } \\
\text { researcher (design, } \\
\text { computer science) }\end{array}$ & $\begin{array}{l}\text { Data collection, } \\
\text { initial coding, } \\
\text { initial themes }\end{array}$ \\
\hline R2 & $\begin{array}{l}\text { Early stage } \\
\text { researcher (design, } \\
\text { computer science) }\end{array}$ & $\begin{array}{l}\text { Collaborative } \\
\text { review of } \\
\text { emerging codes } \\
\text { and themes }\end{array}$ \\
\hline R3 & $\begin{array}{l}\text { Post-doc (design, } \\
\text { computer science, } \\
\text { youth mental health) }\end{array}$ & $\begin{array}{l}\text { Collaborative } \\
\text { review of } \\
\text { emerging codes } \\
\text { and themes }\end{array}$ \\
\hline R4 & $\begin{array}{l}\text { Full professor } \\
\text { (design, computer } \\
\text { science, positive } \\
\text { psychology) }\end{array}$ & $\begin{array}{l}\text { Collaborative } \\
\text { review of } \\
\text { emerging codes } \\
\text { and themes }\end{array}$ \\
\hline
\end{tabular}




\section{Theme 3: Choice and customization}

Concepts 3, 5, 6, and 8 mention functionalities of choice, in terms of deciding what type of content should be focused on (Concept 8) or what media this content should be presented through (Concept 6 ). This theme thus fulfils a range of functions.

Our participants thought that avatar customization would create a stronger bond with the young person, and translate into more engagement and longer use of the app. By being able to customize the avatar, it would become more personal, and also make it easier to establish an emotional connection with the avatar.
Being able to select a type of content was linked by our participants to making the app more useful for diverse groups of young people, in order to accommodate individual differences. Some young people may be more interested in getting information, some may want to do activities - and this further differentiates in terms of what information, which activities.

This theme was also introduced because our participants thought that offering choice and customization would make the technology more useable in diverse situations.

Table 2. Group concepts during British Psychological Society workshop

\begin{tabular}{|c|c|c|}
\hline Group & Name & Description \\
\hline 1 & $\begin{array}{l}\text { Video Log on social media } \\
\text { perils }\end{array}$ & $\begin{array}{l}\text { Aimed at educating young people that not everything they see online is real; young people, and } \\
\text { prominent YouTubers, would provide information on issues around social media, in the form of } \\
\text { short and fun videos embedded into various social media platforms. }\end{array}$ \\
\hline 2 & $\begin{array}{l}\text { App for young people with } \\
\text { diabetes }\end{array}$ & $\begin{array}{l}\text { An app to provide information and motivation for young people with diabetes, to ease the } \\
\text { associated negative impact on mental health. It would offer two-way exchange between young } \\
\text { people and people who function as caregivers in their network, and would also be linked to an } \\
\text { online community. It would have to be presented in an engaging way, as many young people } \\
\text { currently find mental health apps boring. }\end{array}$ \\
\hline \multirow[t]{2}{*}{3} & The real me & $\begin{array}{l}\text { A technology that would offer young people methods for intrapersonal reflection. It would include } \\
\text { compassionate notes to self, things I am good at, daily diary (good and bad), my thoughts and } \\
\text { feelings, what is important to me and what are my values, what helps me cope. Additionally, it } \\
\text { would feature an avatar the young person could customize. }\end{array}$ \\
\hline & & $\begin{array}{l}\text { This would be an opportunity for young people to express, track and save their true self, and } \\
\text { remind themselves of their strengths and values to enhance resilience, stability and identity. }\end{array}$ \\
\hline 4 & Recovering from concussion & $\begin{array}{l}\text { Technology to ease the mental health burden of concussion recovery. It would offer a monitoring } \\
\text { system for symptoms like headaches, dizziness, memory, concentration, fatigue, irritability, } \\
\text { anxiety and depression. It would allow tracking over time, have a journal or log function, and may } \\
\text { offer memory games and cognitive assessments. It would offer ways to visualize symptoms. }\end{array}$ \\
\hline 5 & Coping with bullying app & $\begin{array}{l}\text { An app to increase resilience in the face of bullying. It would have features to identify distress, } \\
\text { offer individual coping strategies, and offer ways to connect with others. Smartphone sensors } \\
\text { would be used to provide biofeedback e.g. for stress. A customizable avatar would be used for } \\
\text { labelling feelings. }\end{array}$ \\
\hline 6 & Sleep app & $\begin{array}{l}\text { An app to improve sleep hygiene. Features embedded would include YouTube videos which } \\
\text { have gone through quality control by experts, and additional information for children and young } \\
\text { people, as well as games, and a panic button to provide information in necessary situations. It } \\
\text { would be interactive, for example if a child is watching YouTube, but it's late then there would be } \\
\text { an alert to go to bed. It would link to a kit with resources, such as YouTube videos, with coping } \\
\text { strategies or another preferred media of the client's choice. All content would be approved and/or } \\
\text { co-produced by professionals. }\end{array}$ \\
\hline 7 & YouTube channel & $\begin{array}{l}\text { A YouTube channel which is run by mental health clinicians and young people, in collaboration } \\
\text { with high profile vloggers and celebrities, where they speak about real life experiences and } \\
\text { provide coping strategies, as well as signposting to additional resources. Channel content and } \\
\text { comments would be moderated by clinicians and young people. }\end{array}$ \\
\hline 8 & Developing resilience & $\begin{array}{l}\text { A technology to develop resilience in young people. It would provide and describe strategies for } \\
\text { coping skills using avatars, and offer a choice of situations and topics. The technology could be } \\
\text { designed by the young person, and they could choose which situation to focus on, e.g. bullying. It } \\
\text { would be solution focused and offer a variety of responses, as well as positive feedback. It would } \\
\text { be critical to: avoid negatives and failures, provide a realistic environment and positive role } \\
\text { models, as well as a range of responses; it would need to be confidential and also provide a } \\
\text { safety net that connects to humans in case of emergencies. }\end{array}$ \\
\hline 9 & Positive Paws & $\begin{array}{l}\text { A virtual pet that would offer positive feedback and encourage physical and mental wellbeing, } \\
\text { comparable to a Tamagotchi with GPS and Snapchat. It would be embedded in a shared } \\
\text { community. It would provide happiness by requiring the user to care for pet health, responsibility } \\
\text { + purpose exercise and thinking of others. It would store a GPS map of shared walks and count } \\
\text { steps on the phone. It would offer pet health tips. It would allow customized pets to be created, } \\
\text { and effects of care would be visible on the pet. The pet would also show positive behavior, like }\end{array}$ \\
\hline
\end{tabular}




\section{Theme 4: Rich interactions}

Concepts 3, 4, 5, 6, and 9 describe technologies which are rich in interactivity. This means the young person using the technology is not a passive recipient of information, as for example in Concept 7 , but would be interacting with the system in diverse ways, including playful interactions in the form of games (Concepts 4 and 6). Our participants thought that offering highly interactive and gamelike mechanics would make these technologies inherently more interesting and fun to use, which would translate into prolonged use and deeper engagement. For technologies which employ some sort of avatar, having a broader range of interactions available with the avatar was suggested to increase the emotional bond with it, for example being able to play with the virtual pet from Concept 9.

In summary, Study 1 confirmed much of what we already saw in the initial literature review on youth media preferences. The data from Study 1, in combination with the initial literature review, point to four strategies to improve fit of mental health technologies with young people:

- Social connectivity

- Use of video

- Tailorability

- Rich interactions

Fully instantiated games, which were strategies identified in our literature review, only got limited consideration during the workshop, which is why we did not include them in the final list.

\section{Study 2: App review}

Following the completion of Study 1, we conducted an explorative review of 29 exemplar youth mental health promotion and prevention apps, looking at how these apps deliver content to young people, and how they can be interacted with.

Mental health apps are complex systems with different aspects that reviewers may decide to pay attention to. We used MM as the primary lens [44]. We had two reasons for this decision: the utilization of MM for youth mental health systems has not been studied up to this point, and modalities are the defining characteristics of the youth media preferences indicated in Related work. Therefore, reviewing MM opened up a new avenue of inquiry and a suitable one for investigate the study question.

After identifying relevant apps (see Data collection and analyses for details), we analyzed them using a general inductive approach. A general inductive approach "is carried out through multiple readings and interpretations of the raw data" [49], building on the premise that continued engagement with data will eventually allow researchers to recognize patterns and interpret them. Open and interpretive as it is, a general inductive approach lends itself well to exploratory reviews and new lines of inquiry. There exists a precedent for using a general inductive approach for youth mental health technology reviews [40].

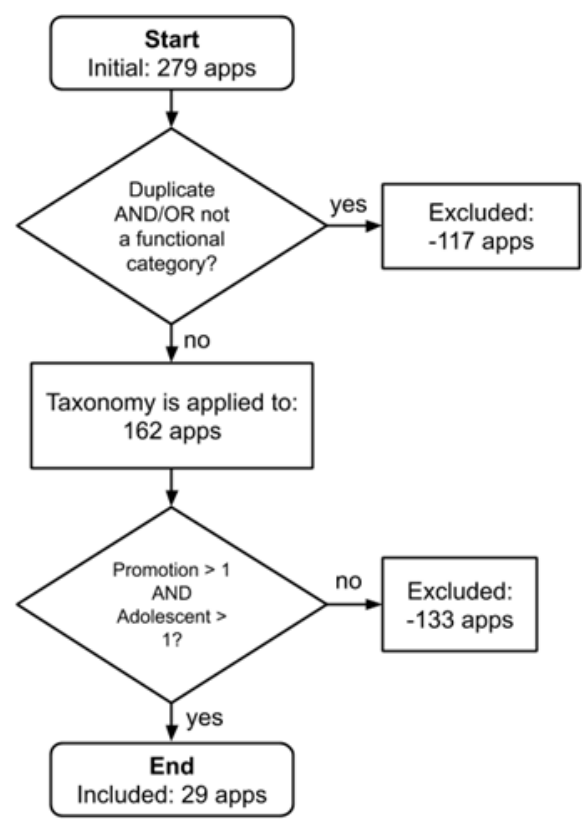

Figure 1. Selection process

\subsection{Data collection and analyses}

To identify apps for review, we decided to use the same avenues which would be accessible to a young person. First, we queried "mental health" both in Google's Play Store and Apple's App Store and noted down all the apps that were suggested to us. Next, we looked up curated app recommendation lists from the prominent youth mental health organizations mentioned previously, like ReachOut [33] and Healthy Young Minds [52] (part of the UK National Health Service). Combined, this led to the identification of 279 entries, including duplicates.

There are many more mental health apps than the 279 we collected initially [53], but both app stores only suggest a small selection when querying. However, this was not an issue, as we did not aim to collect an exhaustive list of all mental 
health apps. Instead, we looked for exemplar apps that could be recommended to a young person, and used them to provide indications for strategies on content delivery. Next, we identified the sub-set of those apps that may be considered youth mental health promotion apps. To achieve this, we developed a set of preliminary criteria and a ratingsystem for sorting these apps according to whom we understood their target audience to be, and what their goal was. We made these decisions based on the visual look of an app, the language used in both the app and the description for the app in its respective store, the type of topics it addressed, and the strategies it used. Decisions were discussed within our team until we reached a consensus. After applying this process, we were left with 29 youth mental health promotion apps (see Table 2 for a list of the included apps; see Fig 3 for an overview of the selection process).

We then created rich descriptions-long-form text explications of functionality-of all 29 apps in terms of what they were aimed at, how interventions were delivered, and what interaction methods were offered. App store descriptions of the apps, screenshots and user-comments served as a foundation; we then installed, manually explored and tested all the apps and took notes.

Finally, one researcher read through the notes looking for content delivery strategies, for example the modalities used, as well as for higher-level strategies, in multiple iterations.

\subsection{Results}

Overall, the apps we reviewed mostly featured minimalistic MM functionality: single-tap touch for input, and static images and text for output. There were some examples of more ambitious technology use, like turning the camera into a heartrate sensor (ReachOut Breathe), but these were rare.

\section{Modalities for input}

Single tap touch was the dominant input modality. This held true not only for the basic commands (which is expected), but even for the few game-like mechanics in our sample, e.g., Happify. Most apps only offered interaction using single tap touch.

Still common, but slightly less so, was the use of written text as input. Superficially, written input could be considered to be simply a derivative of single tap touch, as single tap touch is how written text is produced. We consider it an independent modality, because written text may also be produced using voice on most devices-natively, through the phone's operating system-and also because written text has much more expressive depth than basic single tap touch. Examples of YMH promotion apps allowing text input would be 7 Cups of Tea, Smiling Mind, Booster Buddy, In Hand and Me - My Mental Health.
Three apps offered an option to take pictures as input, e.g., Happify, featuring a picture-based version of the Three Good Things intervention [54].

Two apps featured input modalities which each constituted a category of their own, due to their unusual nature. PAUSE uses a customized version of single tap drag gestures to create an interactive visualization, which aims to calm users down and to reduce anxiety. ReachOut Breathe makes use of the smartphone camera to determine the heart rate of the user. For this, the user has to place one finger on the camera, and the app is then able to pick up the heartrate by recognizing brightness changes caused by blood circulation. 5.3.2 Modalities for output

Written text alongside static images was the prevailing output modality combination and part of all 29 apps. Some apps applied animations to their visualizations, mostly cosmetic, e.g., transitions between screens-however, in rare cases also this strategy was seemingly directed at improving the impact of an intervention. For example, Booster Buddy used an animated anthropomorphic animal buddy, which a user could interact with using touch. When touched, the buddy responded with a positive animation, expressing happiness.

Audio output was part of 11 out of 29 apps (e.g., Headspace). Audio was used both for speech and music, and was mostly present in apps for guided meditation; the only exceptions to this were Recharge, which used audio for an alarm clock function aimed a regulating sleep, and Booster Buddy, which had audio clips playing during the companion's animations.

\section{Higher-level functionality}

This category looks directly at some of the higherlevel strategies which came out of Study 1 for improving fit for young people. These are mostly emerging strategies achieved by combining various modalities.

The apps were strongest with respect to including social connectivity. Some apps offered either access to peer communities, e.g., TalkLife, or access to communication with experts, e.g., 7 Cups. A young person looking to engage with peers or experts will likely be able to locate an app allowing it. Communication was predominantly text-based, especially in group-forum types of conversations where postings are visible to everyone. (There may be reasons in terms of moderating content and safeguarding for restricting visual communication in this context.) 
Table 3. Included apps and mental health strategies

\begin{tabular}{|c|c|}
\hline Name & Mental health strategy \\
\hline 7 Cups: Anxiety \& Stress Chat & Mindfulness, facilitates contact with mental health counsellors \\
\hline Bliss: Harvard Mental Health & Mindfulness meditation \\
\hline Booster Buddy & Habit building and changing, tracking \\
\hline Breakup Shakeup & Recommends activities, facilitates socializing \\
\hline Daylio & Diary writing, tracking \\
\hline Fabulous & Meditation, various activities and exercise \\
\hline FearTools - Anxiety Aid & Mindfulness, CBT, breathing exercises \\
\hline Good Habit Maker & Habit building and changing through reminders \\
\hline HabitBull & Habit building, tracking \\
\hline Happify & Mindfulness meditation, CBT, emotional regulation \\
\hline Headspace & Mindfulness meditation \\
\hline In Hand & Emotional regulation, inspiration quotes, taking pictures \\
\hline Jot Happy for Mental Wellness & Changing mindset, goal setting, safety planning, meditation \\
\hline Kelaa Mental Resilience & Resilience, mindfulness \\
\hline Know Yourself Personality Test & Assessment, articles to read \\
\hline Liebe dich selbst Selbstliebe & Exercises to improve self esteem \\
\hline LoveSmart & Assessment, articles to read \\
\hline Me - My Mental Health & Diary writing, helplines \\
\hline Meditation Music - Relax & Meditation, music \\
\hline MiYo & Tracking with reward systems \\
\hline Noggin Notes & $\begin{array}{l}\text { Motivational quotes, gratitude diary, tracking emotions, blog posts on mental } \\
\text { health }\end{array}$ \\
\hline Pacifica - Stress \& Anxiety & $\begin{array}{l}\text { CBT, mindfulness meditation, guides, mood-, goal- and health-tracker, } \\
\text { journaling, daily challenges, facilitates peer support }\end{array}$ \\
\hline PAUSE & Mindfulness-based drawing exercise \\
\hline Pin It or Bin It & CBT, "pin" good memories, "bin" bad ones \\
\hline ReachOut Breathe & Breathing exercise, bio-feedback (heartrate) \\
\hline ReachOut WorryTime & Writing down worries and crumbling them up when they become obsolete \\
\hline Recharge & Regulates sleep time, encourages physical activity \\
\hline Smiling Mind & Mindfulness meditation \\
\hline TalkLife - You're Not Alone & Facilitates peer support \\
\hline
\end{tabular}

Dynamic content was lacking however. Most apps delivered their content in a static fashion, e.g. text alongside images, without the option to interact with the content. Only Happify made use of a game, and only in a very limited fashion.

The apps we reviewed were weakest with respect to customization and choice. Content presentation was mostly pre-determined and linear, without factoring in choice. Customization never went beyond minimal cosmetic adjustments, e.g., exchanging the icons for an app. Additionally, young people did not get the chance to impact how they would interact with the apps. Most apps relied primarily on single tap touch and did not offer any 
alternatives. The same was true for how content was presented. Even though there was some slight variation between apps, as illustrated for example by guided-meditation apps using voice output, there was never any opportunity for choice with regard to interaction within an app.

In summary, apps used modalities in reduced ways, especially for input which relied heavily on single tap touch. Output was very text-heavy, with static visualizations.

\section{Mental health strategy}

There was some diversity of mental health strategies across the 29 apps, but most of them were realized with a minimal use of MM (see Table 2 for full list).

Eight apps offered Mindfulness [55] exercises, e.g., 7 Cups, making it the most common mental health strategy we found. In some cases, e.g., Headspace, this was combined with guided meditation exercises. Mindfulness exercises predominantly aim to increase a person's awareness and appreciation of the present [55]. If combined with meditation, these activities were usually presented through audio as guided meditation, otherwise they were text-based.

Four apps offered various diary writing exercises, e.g., Daylio. Diary writing exercises aim to increase intrapersonal reflection, to make fleeting and vague thoughts tangible, and to make a person more aware of their intrapersonal and interpersonal resources [56]. These activities were entirely text-based.

Three apps offered exercises from Cognitive Behavior Therapy (CBT) [27], e.g., Happify. CBT exercises predominantly aim to re-contextualize and replace negative automatic thoughts [27]. These activities were entirely text-based.

Three apps facilitated behavior change [57], e.g., Booster Buddy. Behavior change aims to create an upward spiral of positive effects building on the adoption of small, positive acts during the day, or the reduction of harmful acts [57]. These activities were entirely text-based.

\section{Discussion}

Our goal was to explore the fit between youth mental health technologies and young people's preferences for content delivery. We achieved this by investigating strategies for fit through the eyes of youth mental health experts, and by reviewing youth mental health apps for prevention and promotion. The data indicate significant hurdles to the proliferation of youth mental health apps.

\subsection{Principal findings}

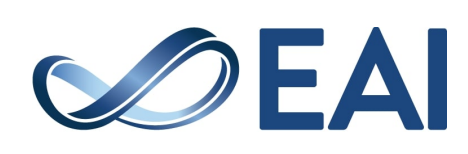

Study 1 shows strategies through which mental health technologies could become engaging for young people, according to youth mental health experts. Study 2 provides a perspective on the current state of mental health apps available to young people. Combined, Study 1 and 2 both indicate a substantial gap between likely effective strategies and youth preferences, i.e., a lack of fit.

Youth mental health promotion apps are not tailorable to any meaningful degree, they are barely interactive, rely heavily on static text, and they do not make meaningful use of visual media. Some apps offer ways for young people to interact with each other, but this too is only in a limited fashion. There may be good safeguarding reasons for limiting social interaction, but at least the other strategies - tailorability, interactivity, use of visuals - could be substantially improved if we are to close the engagement gap and to increase the uptake and effectiveness of youth mental health apps.

The design of these technologies needs to be more closely oriented to what young people are actually interested in, to achieve fit, and also be cognizant of the trajectory of these interests. The increasing pull towards highly interactive, visual, social and tailorable technologies risks - by itselfwidening the gap between what young people expect and what we are providing. By recognizing the importance of this fit and by designing for it, a range of opportunities could open up for improving youth mental health technologies, as outlined in the following sections.

First, however, we will connect our findings with two relevant theoretical models: the process model of engagement, and self-determination theory.

\subsection{Process model of engagement}

The process model of engagement describes engagement as a three-step process: the (i) initial point of engagement leading into (ii) the period of engagement, which again leads into (iii) disengagement-with a potential for reengagement [20]. Criteria for initial engagement include system-sided attributes, like aesthetics and novelty, and person-sided ones, like interest and motivation. The period of engagement also relies on aesthetics, but introduces attributes like control, interactivity, challenge, feedback and interest, which would not be fully perceivable prior to engaging with a system. Finally, disengagement deals with attributes like (lack of) usability, (lack of, or too much) challenge, and interruptions.

The shortcomings we observed - to recap, a lack of fit, manifested as a lack of tailorability, interactivity, visual media, and overuse of static text-all relate to the period of engagement, 
meaning they become relevant after engagement is initiated. We propose that a lack of tailorability most closely relates to the engagement-attribute of control. Lack of interactivity directly relates to the interactivity-attribute. Lack of visual media most closely relates to the aesthetic and sensory appealattribute, in addition to having some possible overlap with the novelty-attribute. Overuse of static text may impact aesthetics and sensory appeal, as well as novelty. To see our findings situated within the Process model of engagement, see fig 4.

The Process model of engagement would predict that if a number of attributes in the period of engagement are lacking, then the consequence would be a quicker move towards disengagement. The shortcomings we discovered align well with the period of engagement phase, making timely disengagement with youth mental health promotion apps likely, i.e., young people would, after initial engagement, quickly stop using mental health promotion apps.

\subsection{Self-determination theory}

Another lens through which to examine our data is self-determination theory (SDT) [58]. SDT states that positive engagement in any environment-be it work, games, or any kind of technology-is predicated on the fulfilment of three fundamental needs: autonomy, relatedness, and competency [59]. The last couple of years have seen a strong push towards applying SDT to the technology context, with studies showing the merits of this approach coined Positive Computing [60].

The strong prevalence of static content we encountered, the generally low levels of interactivity, and missing options to customize the apps we saw would likely mean that these apps do not fare well in terms of offering autonomy and competency. While this would need to be studied separately to make any confident statements, if autonomy and competency are not offered by these apps, then SDT would provide an additional explanatory framework for why young people are not interested in engaging with these technologies.

\subsection{Improving fit}

Despite the specific nature of the interventions not being a selection criterion in our analyses, we still ended up with a fairly homogenous set of interventions across all apps. Some apps used CBT approaches, some mindfulness, some diary writing - all of which rely heavily on language. This may already pre-select to some degree the type of young people who would be willing to engage with such an app. More sophisticated modes of interaction would allow the introduction of less language-prone mental health strategies, and in turn, may achieve fit for more young people, allowing them to benefit from these apps,

One example of a new range of activities made possible through more sophisticated interactions would be art therapy, where visual expression serves as a mechanism for introspection and growth [61], [62]. While art therapy would align itself with the visual preferences we found in young people's use of digital media, almost none of the apps we found used mechanisms aligned with art therapy. Pulse, a meditative drawing app, would be the only potential exception to this, but it was very limited in terms of its range of interactions and possible expressive depth.

Another type of activity that could be opened up are Somaesthetic activities [63], where subtle interactions and intimate, embodied feedback may be used to create meditation and mindfulness experiences [64]. Even though smartphones are technologically able to give subtle physical feedback which would seemingly lend itself to Somaesthetic activities, for example using vibration as feedback during mindfulness exercises [55], [65], this has not yet been put into practice in $\mathrm{YMH}$ promotion apps.

The commonly used language-centered approaches could also have unintended consequences. They favor the analytical over the intuitive, and exclude young people who may not be able to engage with the language of the app at the necessary level. As one example, a group of young people with a high prevalence of mental health problems, and thus with a strong claim on our collective efforts, are refugee youth [66], who, especially in the first critical weeks and months after their arrival, will rarely achieve sufficient mastery of the local language to engage with appinstructed CBT exercises, or any form of languagebased mental health promotion app.

\subsection{Opportunities for research}

Offering different modalities also opens up new opportunities for researchers to better understand the relevant mechanisms leading to positive outcomes from promotion and prevention strategies.

For example, one of the most potent promotion strategies is the gratitude letter [67], where a person writes a letter to someone detailing all the ways in which the person is grateful for their support. This kind of activity could be completed using video instead of writing a letter by hand. A concept around a YouTube channel (as in Study 1, Concept 7) could be used both to deliver the video gratitude letter and open up potential additional benefits through positive community support [68].

As another example, the wellbeing benefits of physical activity are well established [69], [70], but there is no research currently on the potentially 
accumulating benefits of combining more physical activity with a game-based approach utilizing GPS, animation and other approaches to implementing MM (as in Study 1, Concept 9).

Sleep quality mediates wellbeing [71] and there are activities to support sleep quality which use a 'pen-and-paper' type approach [72], but we do not know the potential benefits that may arise if we offer an interactive toolkit of activities to improve sleep quality (as in Study 1, Concept 6).

By observing the degree to which these shifts in modality can impact the effect of prevention and promotion activities, we could learn more about the underlying mechanisms and what part of an activity contributes to its success. Understanding this interplay between modalities and activities, together with fit, may play a significant role in improving the effectiveness of digital mental health interventions in the future.

The current lack of research on mental health apps is disconcerting, with only a handful of studies in spite of 12,000 publicly available mental health apps [53]. Research will never be able to study all the mental health apps which are currently available to the public, at least not to any meaningful degree, especially if the number of apps continues to rise (which is to be expected). However, we did observe, just in our small sample of 29 youth mental health promotion apps, repeating components. A subsequent study could use the lenses of MM and higher-level components to arrive at a taxonomy of mental health app building blocks. This would reduce the need to study 12,000 full apps and necessitate the study of, at most, a few dozen building blocks instead. Understanding the impact of individual components would allow us to predict- to some degree - the merits of any new app, based on looking at its component parts, and connecting them with existing research, instead of treating every app like an entirely new unknown object. This could also offer a potential solution to the impossibility of conducting an RCT for every marginally different new mental health app.

Finally, as mentioned earlier, mental health research is increasingly shifting from asking "Does this work?" to "What aspect of this work for whom?" [31]. This more granular question highlights the importance of researching the design of mental health technologies on a more modular level to allow meaningful improvements in the future. The currently widespread approach of treating mental health technology as a black box and giving the same black box to all people to achieve some effect, without understanding how this effect came to be, will not be sufficient anymore. Tailorable and personalized mental health technologies will be enabled by modular, component-based research.

\subsection{Limitations}

There was a specific limitation in this study due to our chosen process of deciding which apps to include in our review. Most apps did not make their target audience or their purpose explicitly clear. We took measures to ensure that we decided consistently, but other researchers may have included apps we did not include, and vice versa. There is, as of yet, no standardized classification metric for mental health apps that could have supported this process; this is a gap that should be addressed.

A further limitation is in not including young people in the workshop for Study 1. We touched on our reasons for this briefly in Section 4. In future work, there is a need to include young people in the design of mental health technologies; in fact, we consider the input from young people critical if we are to design engaging mental health technologies. This further work would complement Study 1, where the voice of youth was included indirectly through our expert participants functioning as surrogates for the young people they have had experience with. We plan to conduct workshops with young people in the immediate subsequent studies.

\section{Conclusion}

We investigated the relationship between youth technology preferences-from the perspective of existing research, and through the eyes of youth mental health experts - and youth mental health promotion apps, by reviewing a set of available apps for prevention and promotion. Our results indicate a mismatch: the apps did not appear to align with young people's preferences. Instead, young people are presented with a myriad of apps with minimal functionality.

Future work should establish a taxonomy of mental health app components and modalities, study their impact in terms of their efficacy as mental health activities, and map how they influence young people's engagement with and interest in using these technologies. Future work should also engage directly with youth to design the next generation of mental health promotion technologies and to deliver on their inherent potential.

\section{Acknowledgements}

Our research has been funded by the European Union's Horizon 2020 research and innovation programme under the Marie Skłodowska-Curie grant agreement No. 722561. 


\section{References}

[1] The Children's Society, "The Good Childhood Report 2017," The Children's Society, 2017. [Online]. Available:

https://www.childrenssociety.org.uk/sites/default/fi les/the-good-childhood-report-2017_fullreport_0.pdf.

[2] World Health Organisation, "Adolescence: a period needing special attention." [Online]. Available:

http://apps.who.int/adolescent/seconddecade/section2/page1/recognizingadolescence.html. [Accessed: 13-Jan-2019].

[3] R. C. Kessler, P. Berglund, O. Demler, R. Jin, K. R. Merikangas, and E. E. Walters, "Lifetime prevalence and age-of-onset distributions of DSMIV disorders in the National Comorbidity Survey Replication," Arch. Gen. Psychiatry, vol. 62, no. 6, pp. 593-602, Jun. 2005.

[4] NHS Mental Health Task Force, "The Five Year Forward View for Mental Health," Mar. 2018. [Online]. Available:

https://www.england.nhs.uk/wpcontent/uploads/2016/02/Mental-Health-TaskforceFYFV-final.pdf.

[5] I. Wolfe, A. Macfarlane, A. Donkin, M. Marmot, and R. Viner, "Why children die: death in infants, children, and young people in the UKPart A," Royal College of Paediatrics and Child Health, 2014.

[6] Wahlbeck Kristian, "Public mental health: the time is ripe for translation of evidence into practice," World Psychiatry, vol. 14, no. 1, pp. 3642, Feb. 2015.

[7] D. J. Rickwood, F. P. Deane, and C. J. Wilson, "When and how do young people seek professional help for mental health problems?," Medical Journal of Australia, vol. 187, no. 7, p. S35, 2007.

[8] E. Frith, "Access and waiting time in children and young people's mental health services." [Online]. Available:

https://epi.org.uk/publications-and-research/accessto-camhs-2018/.

[9] V. Patel et al., "The Lancet Commission on global mental health and sustainable development," The Lancet, vol. 392, no. 10157, pp. 1553-1598, Oct. 2018.

[10] D. M. Hilty, D. C. Ferrer, M. B. Parish, B. Johnston, E. J. Callahan, and P. M. Yellowlees, "The Effectiveness of Telemental Health: A 2013 Review," Telemedicine and e-Health, vol. 19, no. 6, pp. 444-454, May 2013.

[11] D. Rickwood, M. Webb, V. Kennedy, and N. Telford, "Who Are the Young People Choosing Web-based Mental Health Support? Findings from the Implementation of Australia's National Webbased Youth Mental Health Service," JMIR Ment Health, vol. 3, no. 3, p. e40, Aug. 2016.
[12] T. M. Fleming et al., "Maximizing the Impact of e-Therapy and Serious Gaming: Time for a Paradigm Shift," Front. Psychiatry, vol. 7, 2016.

[13] H. Christensen, J. Reynolds, and K. M. Griffiths, "The use of e-health applications for anxiety and depression in young people: challenges and solutions," Early Interv Psychiatry, vol. 5 Suppl 1, pp. 58-62, Feb. 2011.

[14] F. H. McKay, A. Wright, J. Shill, H. Stephens, and M. Uccellini, "Using Health and Well-Being Apps for Behavior Change: A Systematic Search and Rating of Apps," JMIR mHealth and uHealth, vol. 7, no. 7, p. e11926, 2019.

[15] S. Prince-Embury, "Three-Factor Model of Personal Resiliency and Related Interventions," in Resilience Interventions for Youth in Diverse Populations, Springer, New York, NY, 2014, pp. 25-57.

[16] S. Lyubomirsky and K. Layous, "How Do Simple Positive Activities Increase Well-Being?," Current Directions in Psychological Science, vol. 22, no. 1, pp. 57-62, Feb. 2013.

[17] S. M. Schueller, "Person-Activity Fit in Positive Psychological Interventions," in The Wiley Blackwell Handbook of Positive Psychological Interventions, John Wiley \& Sons, Ltd, 2014, pp. 385-402.

[18] R. B. Thompson, C. Peura, and W. F. Gayton, "Gender differences in the person-activity fit for positive psychology interventions," The Journal of Positive Psychology, vol. 10, no. 2, pp. 179-183, Mar. 2015.

[19] E. G. Lattie, E. C. Adkins, N. Winquist, C. Stiles-Shields, Q. E. Wafford, and A. K. Graham, "Digital Mental Health Interventions for Depression, Anxiety, and Enhancement of Psychological Well-Being Among College Students: Systematic Review," Journal of Medical Internet Research, vol. 21, no. 7, p. e12869, 2019.

[20] H. L. O'Brien and E. G. Toms, "What is user engagement? A conceptual framework for defining user engagement with technology," Journal of the American Society for Information Science and Technology, vol. 59, no. 6, pp. 938955,2008

[21] B. Laurel, Computers As Theatre. Boston, MA, USA: Addison-Wesley Longman Publishing Co., Inc., 1991.

[22] C. Peterson, A Primer in Positive Psychology. Oxford University Press, USA, 2006.

[23] M. E. P. Seligman and M. Csikszentmihalyi, "Positive Psychology: An Introduction," in Flow and the Foundations of Positive Psychology, Springer, Dordrecht, 2014, pp. 279-298.

[24] A. Edmondson, "Framing for Learning: Lessons in Successful Technology Implementation," in Fundamentals of Organization 
Development, 4 vols., London: SAGE Publications Ltd, 2010, pp. IV121-IV121.

[25] A. Horgan and J. Sweeney, "Young students' use of the Internet for mental health information and support," J Psychiatr Ment Health Nurs, vol. 17, no. 2, pp. 117-123, Mar. 2010.

[26] F. K. Arnberg, S. J. Linton, M. Hultcrantz, E. Heintz, and U. Jonsson, "Internet-delivered psychological treatments for mood and anxiety disorders: a systematic review of their efficacy, safety, and cost-effectiveness," PLoS ONE, vol. 9, no. 5, p. e98118, 2014.

[27] D. D. Ebert et al., "Internet and computerbased cognitive behavioral therapy for anxiety and depression in youth: a meta-analysis of randomized controlled outcome trials," PLOS ONE, vol. 10, no. 3, p. e0119895, 2015.

[28] L. Wozney et al., "RE-AIMing e-Mental Health: A Rapid Review of Current Research," 2017. [Online]. Available:

https://www.nweurope.eu/media/3970/emhliterature-review_final-en.pdf.

[29] T. Donker, K. Petrie, J. Proudfoot, J. Clarke, M.-R. Birch, and H. Christensen, "Smartphones for Smarter Delivery of Mental Health Programs: A Systematic Review," J Med Internet Res, vol. 15, no. 11, Nov. 2013.

[30] R. Grist, J. Porter, and P. Stallard, "Mental Health Mobile Apps for Preadolescents and Adolescents: A Systematic Review," Journal of Medical Internet Research, vol. 19, no. 5, p. e176, 2017.

[31] D. Bakker, N. Kazantzis, D. Rickwood, and N. Rickard, "Mental Health Smartphone Apps: Review and Evidence-Based Recommendations for Future Developments," JMIR Ment Health, vol. 3, no. 1, Mar. 2016

[32] Anxiety and Depression Association of America, "Reviewed Mental Health Apps." [Online]. Available: https://adaa.org/findinghelp/mobile-apps. [Accessed: 15-Jul-2019].

[33] ReachOut Australia, "Tools and Apps." [Online]. Available: https://au.reachout.com/toolsand-apps. [Accessed: 04-Jan-2018].

[34] S. Lyubomirsky, The how of happiness: A scientific approach to getting the life you want. New York, NY, US: Penguin Press, 2007.

[35] K. Layous and S. Lyubomirsky, "The How, Why, What, When, and Who of Happiness," in Positive Emotion, J. Gruber and J. T. Moskowitz, Eds. Oxford University Press, 2014, pp. 472-495.

[36] Ofcom, "Children and Parents: Media Use and Attitudes Report 2017," p. 306.

[37] "Gaming penetration among children in the U.S. 2015 | Statistic," Statista. [Online].

Available:

https://www.statista.com/statistics/274600/gamingpenetration-among-children-in-the-us/. [Accessed: 13-Jan-2019].
[38] "U.S. most popular video game genres 2017," Statista. [Online]. Available:

https://www.statista.com/statistics/189592/breakdo wn-of-us-video-game-sales-2009-by-genre/.

[Accessed: 19-Jul-2019].

[39] K. M. Boydell, M. Hodgins, A.

Pignatiello, J. Teshima, H. Edwards, and D. Willis, "Using Technology to Deliver Mental Health Services to Children and Youth: A Scoping Review," J Can Acad Child Adolesc Psychiatry, vol. 23, no. 2, pp. 87-99, May 2014.

[40] T. Fleming, M. Lucassen, K. Stasiak, M. Shepherd, and S. Merry, "The impact and utility of computerised therapy for educationally alienated teenagers: The views of adolescents who participated in an alternative education-based trial: Adolescents views of computerised therapy," Clinical Psychologist, vol. 20, no. 2, pp. 94-102, Jul. 2016.

[41] S. Oviatt, B. Schuller, P. R. Cohen, D. Sonntag, G. Potamianos, and A. Krüger, Eds., The Handbook of Multimodal-Multisensor Interfaces: Foundations, User Modeling, and Common Modality Combinations - Volume 1. New York, NY, USA: Association for Computing Machinery and Morgan \& Claypool, 2017.

[42] N. O. Bernsen and L. Dybkjær, Multimodal usability. Berlin; New York: Springer, 2009.

[43] S. A. Chhabria and R. V. Dharaskar, "Multimodal Interfaces for Disabled Persons," IJCSC, vol. 2, no. 1, pp. 223-228, Jun. 2011.

[44] E. Freeman, G. Wilson, D.-B. Vo, A. Ng, I. Politis, and S. Brewster, "The Handbook of Multimodal-Multisensor Interfaces," S. Oviatt, B. Schuller, P. R. Cohen, D. Sonntag, G. Potamianos, and A. Krüger, Eds. New York, NY, USA: Association for Computing Machinery and Morgan \& Claypool, 2017, pp. 277-317.

[45] N. Shen et al., "Finding a Depression App: A Review and Content Analysis of the Depression App Marketplace," JMIR mHealth and uHealth, vol. 3, no. 1, p. e16, 2015.

[46] L. S. Clark, "Critical Theory and Constructivism." [Online]. Available: https://www.ihrcs.ch/?p=92.

[47] E. G. Guba and Y. S. Lincoln, "Competing paradigms in qualitative research," 1994. [Online]. Available: https://pdfs.semanticscholar.org/f4ee/6f7b09f4b1c9 943cc36a8aa5a6391e1a92cf.pdf.

[48] V. Braun and V. Clarke, "Using thematic analysis in psychology," Qualitative Research in Psychology, vol. 3, no. 2, pp. 77-101, Jan. 2006.

[49] D. R. Thomas, “A General Inductive Approach for Analyzing Qualitative Evaluation Data," American Journal of Evaluation, vol. 27, no. 2, pp. 237-246, Jun. 2006.

[50] T. Fleming et al., "The Importance of User Segmentation for Designing Digital Therapy 
for Adolescent Mental Health: Findings From Scoping Processes," JMIR Mental Health, vol. 6, no. 5, p. e12656, 2019.

[51] J. Nicholas, A. S. Fogarty, K. Boydell, and H. Christensen, "The Reviews Are in: A Qualitative Content Analysis of Consumer Perspectives on Apps for Bipolar Disorder," Journal of Medical Internet Research, vol. 19, no. 4, p. e105, 2017.

[52] "Healthy Young Minds." [Online]. Available:

https://healthyyoungmindspennine.nhs.uk/. [Accessed: 08-Apr-2019].

[53] C. Chen et al., "Making Sense of Mobile Health Data: An Open Architecture to Improve Individual- and Population-Level Health," Journal of Medical Internet Research, vol. 14, no. 4, p. e112, 2012.

[54] "Three Good Things | Practice | Greater Good in Action." [Online]. Available:

https:/ggia.berkeley.edu/practice/three-goodthings. [Accessed: 15-Apr-2019].

[55] I. Plaza, M. M. P. Demarzo, P. HerreraMercadal, and J. García-Campayo, "MindfulnessBased Mobile Applications: Literature Review and Analysis of Current Features," JMIR Mhealth Uhealth, vol. 1, no. 2, Nov. 2013.

[56] C. Reiter and G. Wilz, "Resource diary: A positive writing intervention for promoting wellbeing and preventing depression in adolescence," The Journal of Positive Psychology, vol. 11, no. 1, pp. 99-108, Jan. 2016.

[57] P. V. Cappellen, E. L. Rice, L. I. Catalino, and B. L. Fredrickson, "Positive affective processes underlie positive health behaviour change," Psychology \& Health, vol. 33, no. 1, pp. 77-97, Jan. 2018.

[58] R. M. Ryan and E. L. Deci, "Selfdetermination theory and the facilitation of intrinsic motivation, social development, and well-being," Am Psychol, vol. 55, no. 1, pp. 68-78, Jan. 2000.

[59] R. M. Ryan and E. L. Deci, SelfDetermination Theory: Basic Psychological Needs in Motivation, Development, and Wellness.

Guilford Publications, 2017.

[60] R. A. Calvo and D. Peters, Positive Computing: Technology for Wellbeing and Human Potential. MIT Press, 2014.

[61] V. Appleton, "Avenues of Hope: Art Therapy and the Resolution of Trauma," Art Therapy, vol. 18, no. 1, pp. 6-13, Jan. 2001.
[62] B. J. Davis, Mindful art therapy a foundation for practice. London: Jessica Kingsley Publishers, 2015.

[63] K. Höök, M. P. Jonsson, A. Stla ahl, and J. Mercurio, "Somaesthetic Appreciation Design," in Proceedings of the 2016 CHI Conference on Human Factors in Computing Systems, New York, NY, USA, 2016, pp. 3131-3142.

[64] R. Shusterman, Body Consciousness: A Philosophy of Mindfulness and Somaesthetics. Cambridge University Press, 2008.

[65] Headspace Inc, Headspace: Guided Meditation \& Mindfulness. Headspace, Inc., 2017.

[66] M. Heeren, L. Wittmann, U. Ehlert, U. Schnyder, T. Maier, and J. Müller, "Psychopathology and resident status - comparing asylum seekers, refugees, illegal migrants, labor migrants, and residents," Comprehensive Psychiatry, vol. 55, no. 4, pp. 818-825, May 2014.

[67] S. Toepfer and K. Walker, "Letters of Gratitude: Improving Well-Being through Expressive Writing.," Journal of Writing Research, vol. 1, no. 3, pp. 181-198, Nov. 2009.

[68] B. Nowell and N. Boyd, "Viewing community as responsibility as well as resource: deconstructing the theoretical roots of psychological sense of community," Journal of Community Psychology, vol. 38, no. 7, pp. 828$841,2010$.

[69] S. J. H. Biddle and M. Asare, "Physical activity and mental health in children and adolescents: a review of reviews," British Journal of Sports Medicine, vol. 45, no. 11, pp. 886-895, Sep. 2011.

[70] P. A. Linley and S. Joseph, "Physical activity: Positive psychology in motion," Positive psychology in practice, pp. 146-164, 2004.

[71] A. J. Howell, N. L. Digdon, K. Buro, and A. R. Sheptycki, "Relations among mindfulness, well-being, and sleep," Personality and Individual Differences, vol. 45, no. 8, pp. 773-777, Dec. 2008.

[72] U. R. Hülsheger, A. Feinholdt, and A. Nübold, "A low-dose mindfulness intervention and recovery from work: Effects on psychological detachment, sleep quality, and sleep duration," Journal of Occupational and Organizational Psychology, vol. 88, no. 3, pp. 464-489, 2015. 